La Comprensión del Concepto de Derechos Humanos en los Niños entre 6 y 14 Años.

Understanding the Concept of Human Rights in Children between 6 and 14 years.

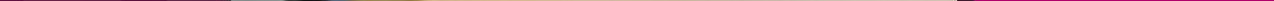




\title{
La Comprensión del Concepto de Derechos Humanos en los Niños entre 6 y 14 Años. ${ }^{1}$ Understanding the Concept of Human Rights in Children between 6 and 14 years.
}

\author{
Jacqueline Benavides Delgado ${ }^{2}$ \\ Universidad Cooperativa de Colombia, Bogota, Colombia
}

Artículo recibido en Marzo de 2016; artículo aceptado en mayo de 2016.

Citación del artículo: Benavides-Delgado, J. (2016). La Comprensión del Concepto de Derechos Humanos en los Niños entre 6 y 14 Años. I+D Revista de Investigaciones, 7(1), 6-13.

\begin{abstract}
Resumen
Desde la declaración de los derechos de los niños en 1959, éstos adquirieron el estatus de sujetos objeto de derecho. Este logro ha permitido a los gobiernos y a las instituciones sociales proteger a los niños y trabajar por su bienestar. El presente estudio se centró en la pregunta ¿Qué comprensión tienen los niños de sus propios derechos? La investigación se realizó en Bogotá con 55 niños escolarizados, entre los 6 y 14 años, de dos estratos socioeconómicos: bajo y alto. Los resultados permitieron concluir que la edad y el desarrollo cognitivo, influyen en la comprensión del concepto de derechos de tal manera que a mayor edad, mayor comprensión y mayor capacidad crítica ante la violación de los mismos. Estos resultados apoyan la teoría de Piaget (1932) acerca del desarrollo cognitivo y moral de los niños. Asimismo, permite reflexionar acerca de la importancia de difundir los derechos de los niños, en contextos escolares, tomando en cuenta el desarrollo cognitivo y moral.
\end{abstract}

Palabras Clave: Psicología del desarrollo, desarrollo del niño, desarrollo mental, pensamiento crítico.

\section{Abstract}

Children are subjects of rights since the declaration of the rights of children in 1969, after World War II. This achievement has enabled assigned to the convention on the rights of children, defend and provide small, a dignified life and ensure their protection governments. What understanding has children of their own rights? This was the question that guided the study in Bogotá with 55 school children, aged between 6 and 14 years. The results concluded that age and cognitive development, influence the understanding of the concept of rights in such a way that the older, more understanding and more critical capacity to the violation thereof. These results support the theory of Piaget on cognitive and moral development of children. They also allow reflection on the importance of disseminating the rights of children in school contexts, taking into account the cognitive and moral development.

Key words: Developmental psychology, child development, mental development, thought developmental psychology, child development, mental development, thought

\section{Introducción}

La infancia como un problema que preocupa a los gobiernos y a la sociedad en general, tiene aproximadamente un siglo de existencia (Takanishi, 1978). Autores como Ariès (1987) han demostrado que durante el siglo XV los niños entre 6 y 7 años eran considerados adultos con derechos y deberes (Benavides, 2015). Así, se les trataba como adultos de tamaño reducido, o como adultos en miniatura. La inclusión de los niños como fuerza de trabajo, los maltratos continuos y su desprotección, han sido recurrentes a lo largo de la historia de la humanidad.

La protección infantil, que resulta ser una práctica cotidiana en el siglo XXI, ha sido un logro de los movimientos sociales consolidados en el siglo XIX. Estos movimientos lucharon por salvar al niño y asegurarle salud

${ }^{1}$ Artículo empírico, enfoque mixto resultado de un proyecto de investigación realizado en el año 2004-2005 en el marco de la línea de investigación de Maltrato Infantil y Violencia Intrafamiliar de la Universidad de los Andes. De la ciudad de Bogotá (Colombia). Dirección Kra. 1E 18ª-10, PBX: 3324365. Fecha de inicio: Enero 2004 fecha de terminación Diciembre 2005.

${ }^{2}$ Psicóloga Universidad de los Andes. Máster en Protección Infantil Universidad del País Vasco. Doctora en Psicología Universidad del Valle-Colombia. Investigadora del grupo Boulumai Universidad Cooperativa de Colombia de la ciudad de Bogotá (Colombia). Dirección calle 172-9- 90 PBX: 33235 65. Correo electrónico institucional: jaqueline.benavidesd@campusucc.edu.co 
y bienestar. De éste modo, como lo afirma Hart (1991), tanto las instituciones privadas como las religiosas y el estado comenzaron a intervenir para proteger a los niños.

Ya en el siglo XX, una consecuencia de los horrores de la primera guerra mundial, fue la redacción, en Ginebra, de la Declaración de los Derechos de los niños de 1924, como un gesto inicial que señalaba la importancia de proteger al niño y brindarle cuidados y atención básica. Sin embargo, fue en la década de los 50 en el siglo XX, donde se iniciaron las acciones humanitarias y se crearon las primeras instituciones internacionales dirigidas a favorecer la protección legal de los niños. En 1959 la Organización de Naciones Unidas (ONU) realizó la Declaración de los Derechos de los Niños. Este documento incluye el derecho a tener un nombre y una nacionalidad, a la igualdad sin distinción de raza, religión, idioma, sexo, nacionalidad u opinión política, a tener una protección especial para el desarrollo físico, mental y social, a una alimentación, vivienda y atención médica adecuada, a una educación y un tratamiento especial para aquellos niños que sufren alguna discapacidad mental o física, a la comprensión y al amor de los padres y de la sociedad, a realizar actividades recreativas y a una educación gratuita, a estar entre los primeros en recibir ayuda en cualquier circunstancia, a la protección contra cualquier forma de abandono, crueldad y explotación y a ser criado con un espíritu de comprensión sexual y tolerancia (WHO, 2014).

El concepto de derecho en el caso de los niños está relacionado con dos elementos fundamentales, por una parte la disyuntiva entre sujeto versus propiedad y por otro lado, el énfasis en las necesidades que todos los individuos tienen por el hecho intrínseco de ser humanos (Hart, 1991). Algunos autores han considerado la existencia de dos posturas respecto a la protección de los derechos de los niños. La primera, y más conocida, es la actitud proteccionista que suple las necesidades básicas de los niños sin consultarles. La segunda una actitud auto determinista que asumen la posibilidad de preguntar a los niños acerca de sus necesidades y forma de suplirlas (Rogers \& Wrightsman, 1978).

Worsfold (1974), considera que históricamente los niños han sido tratados desde una postura paternalista y su conducta ha sido controlada por los padres o por otros adultos. La concepción de los niños que rige esta postura se basa en la idea de niñez como una etapa en la cual existe una total incapacidad de tomar decisiones racionales por tratarse de niños que desean una gratificación inmediata. Este mismo autor expone una visión de Rawls, quien considera como importante y fundamental escuchar las opiniones de los niños acerca de las decisiones que los afectan y enseñarles a tomar decisiones, contemplando su punto de vista. Incluso Rawls (1972) añade: "la intervención paterna debe justificarse por un evidente fracaso o ausencia de razón o deseo" (Worsfold 1974, p. 155). Esta polémica que lleva a plantear un movimiento de "liberación infantil" ha tenido muchas críticas que se basan en el conocimiento del desarrollo infantil y en los beneficios de la autoridad paterna sin pasar al autoritarismo (Baumrind, 1978).

En este campo, como lo señala Hart (1991), la psicología tiene que aportar conocimientos que permitan determinar si la postura auto determinista se podría o no aplicar en los niños que aún no han llegado a la adolescencia. Este primer aporte inicia por la evaluación de la comprensión del concepto de derechos a través de diferentes etapas de desarrollo. En general, los estudios realizados por otras disciplinas, diferentes a la psicología, suelen centrarse en descripciones minuciosas de la violación de los derechos de los niños y se convierten en documentos fundamentales para instaurar políticas públicas y programas de atención a las necesidades de éstos (Donova. Oñate, bravo \& Rivera, 2008; WHO, 2014). Sin embargo, la psicología ha abordado el problema de la comprensión de los derechos, por parte de los niños. Importantes aportes en este campo ha realizado Juan Delval con sus estudios en España. Este autor ha estudiado la comprensión que tienen los niños de sus propios derechos y los resultados obtenidos en el estudio permitieron influir en la formación en derechos en el ámbito escolar y social. La metodología empleada por el autor consistió en la realización de 15 historias que hacían referencia a los diferentes derechos. El equipo de investigadores, dirigido por Delval, realizó un pilotaje aplicando las historias a niños de diferentes edades. Finalmente, este pilotaje arrojó un resultado de 8 historias seleccionadas. Estas historietas fueron aplicadas a niños entre los 8 y 16 años de estrato socio-económico medio. Posteriormente, se tomaron 10 sujetos de cada edad. Melton (1980) también investigó acerca del concepto que tienen los niños de sus derechos. Su investigación se basó en la categorización de las respuestas de 80 niños en tres categorías de pensamiento respecto a sus derechos:

- El primer nivel de pensamiento estaría centrado en una visión de beneficencia en la cual los derechos dependen de que una autoridad los otorgue.

El segundo nivel de pensamiento abarcaría las respuestas que tienen relación con los derechos como privilegios que algunas personas tienen por su estatus o por su edad.

- El tercer nivel consideraría los derechos como parte de la dignidad humana basados en principios de privacidad, libertad, dignidad.

Melton (1980) por su parte encontró que el nivel social y el nivel de escolaridad afectan las concepciones acerca de los derechos. Los niños mayores tienden a plantear el concepto de derecho desde una perspectiva de privilegios más que como beneficios otorgados por una autoridad. De igual forma los niveles de comprensión del concepto de derecho están asociados al estrato social: los niños de estratos altos adquieren el concepto de derecho como privilegios a una edad menor que los de estrato bajo. En 
Colombia, Benavides y Miranda (1997) realizaron un estudio comparando a los niños con historia de maltrato que estaban institucionalizados y aquellos niños de la misma edad, no institucionalizados. Los resultados mostraron que los niños no institucionalizados entre 7-9 años no tenían un concepto claro de los derechos; sin embargo, los niños institucionalizados de estas mismas edades sí podían definir el concepto de derechos con mayor claridad. De otro lado, este estudio aportó otro elemento interesante al encontrar que los niños víctimas de maltrato y que han sido institucionalizados por este motivo, no logran en su mayoría tener un pensamiento crítico hacia los padres mal tratantes cuando la falta cometida por el niño ha sido grave. Por el contrario, si la falta ha sido leve, el razonamiento alrededor de este hecho es diferente.

\section{Perspectiva Piagetiana}

Piaget (1932) plantea un abordaje del desarrollo moral de los niños donde considera dos tipos de moralidad: una heterónoma y otra autónoma. La primera hace alusión a una moral determinada por los otros, que surge en los niños en los primeros años y que va modificándose en la etapa de operaciones concretas $\mathrm{y}$, en la etapa de operaciones formales, se afianza la moral autónoma. "Piaget encuentra que hasta los 6 ó 7 años los niños consideran que lo justo es lo que el adulto ordena y en casos de conflicto entre la obediencia o la justicia prefieren la obediencia" (Villegas de Posada, 1998, p.226). Las primeras nociones de justicia comienzan con una sanción expiatoria, donde el culpable debe sentir dolor proporcional a la falta cometida. Posteriormente, ya en la etapa de autonomía se habla de reparación, más que de expiación de la falta.

Con base en estos planteamientos la presente investigación busca analizar la comprensión del concepto de derecho en niños entre 6 y 14 años y su relación con el desarrollo moral según Piaget (1932).

\section{Objetivos}

- Conocer la comprensión que tienen los niños de 6 a 14 años sobre el concepto de derecho.

- Determinar los mecanismos que los niños conocen para exigir sus derechos

- Conocer la capacidad de los niños para criticar y cuestionar la violación del derecho a no ser víctima de maltrato y abuso por parte de los padres.

\section{Método}

\section{Participantes}

Una muestra de 55 niños (40 niñas y 15 niños), con edades entre los 6 y 14 años. 20 de estrato alto y 35 de estrato bajo. Todos los niños estaban escolarizados (Ver Tabla 1).
Tabla 1.

Descripción de la muestra por edad

\begin{tabular}{ccc}
\hline $6-8$ años & $9-10$ años & $12-14$ años \\
8 niños & 4 niños & 3 niños \\
22 niñas & 15 niñas & 3 niñas \\
30 niños & 19 niños & 6 niños \\
\hline
\end{tabular}

Fuente:Los autores

\section{Instrumentos}

Delval (1995) utilizó historietas en su investigación como una forma de obtener respuesta de los niños. En esta investigación se crearon 2 historietas que fueron puestas a prueba en el pilotaje (Anexo A). Se utilizaron grabaciones de audio en las entrevistas.

\section{Procedimiento}

Se seleccionaron dos instituciones educativas de la ciudad de Bogotá. Una de clase alta y otra de clase social baja. En un primer encuentro se solicitó permiso a las directivas de cada institución y posteriormente se procedió a enviar a los padres los consentimientos que autorizaban a los niños a participar en el estudio. 55 niños fueron entrevistados por psicólogos en cada uno de los centros educativos, siguiendo el protocolo de entrevistas. En la primera parte de la entrevista se creaba un clima de rapport con el niño. Posteriormente se contaban las historietas diseñadas de acuerdo a la edad (Ver Anexo A) En algunos casos se emplearon gráficas para hacer más agradables las sesiones de entrevistas. Después de la lectura de las historietas se le preguntaba al niño si había comprendido y se le pedía que relatara lo que había entendido con el fin de determinar si se hacía las siguientes preguntas:

¿Tú crees que los papás podían haber actuado de otra manera?

¿Tú crees que Pepita o Juan se merecían el castigo?

¿Hay alguien que pueda prohibirles a los padres golpear a sus hijos?

¿Los niños pueden hacer algo para que los papás no les peguen?

¿Sabes tú que es tener derecho a algo?

¿Has oído hablar de los derechos de los niños?

¿Cuáles son?

¿Cuáles son los más importantes?

¿Cómo puedes tu pedir que se te respeten esos derechos?

\section{Resultados}

Las entrevistas fueron grabadas y transcritas en su totalidad; posteriormente se comenzaron a clasificar las respuestas de los niños con un método de triangulación donde tres investigadores leían las respuestas y proponían una categoría. Las categorías resultantes fueron:

a) Juicio crítico

b) Autoridad de los padres 
c) Cuestionamiento de la autoridad paterna

d) Castigos

e) Derechos

f) Clasificación del concepto

g) Derechos recordados

h) Ranking de derechos

\section{a) Juicio Crítico}

Tomando como referencia la muestra completa, se encontró que el $54 \%$ de los niños entrevistados no justificaron el maltrato, ni aceptaban bajo ninguna condición que los niños fueran agredidos por los padres como forma de crianza. El 32\% rechazó el maltrato en primera instancia, sin embargo, al contemplar la gravedad de los actos de los niños, lo justificaron. Asimismo el 9\% aceptaron, sin cuestionar, el maltrato hacia los niños justificándolos por la gravedad de los actos y un porcentaje reducido del $3.6 \%$, aceptó el maltrato independientemente de los hechos.

El análisis realizado por edades, demostró que el 72.4\% de los niños de 6-8 años no justificaban el maltrato. Sólo un porcentaje del $27.6 \%$ justifican el maltrato por la gravedad de los actos cometidos por los niños.

Respecto a la clase social, se encontró que el 100\% de los niños de 6 años, el $80 \%$ de los de 7 y el $66 \%$ de los de 8 años, no justificaron el maltrato dirigido a los niños por parte de sus padres. Respecto a las clases bajas se encontró que el $88 \%$ de los niños de 8 años, el 55\% de los 7 y el 100\% de los de 6 no justificaron el maltrato hacia los niños. En los niños de 9-11 años 68.4\% no justifican el castigo físico. El $15.8 \%$ que lo justifican, lo hacían por la gravedad de los actos cometidos y el $10.5 \%$ que lo justifican abiertamente por la gravedad de los actos, ver Tabla 2.

Tabla 2

Distribución de porcentajes según edad

\begin{tabular}{cccc} 
Edad & $\begin{array}{c}\text { No justifican } \\
\text { el maltrato }\end{array}$ & $\begin{array}{c}\text { Justifican el } \\
\text { maltrato por } \\
\text { la gravedad } \\
\text { de los actos }\end{array}$ & $\begin{array}{c}\text { Justifican el } \\
\text { maltrato sin } \\
\text { tener en } \\
\text { cuenta la } \\
\text { gravedad }\end{array}$ \\
$6-8$ & $72.4 \%$ & $27.6 \%$ & --- \\
$9-11$ & $68.4 \%$ & $15.8 \%$ & $10.5 \%$ \\
$12-14$ & $83.3 \%$ & $16.7 \%$ & ---- \\
\hline
\end{tabular}

Fuente: Los autores

\section{b) Autoridadpaterna}

Respecto a la autoridad de los padres, el 50\% la cuestionan. El $30 \%$ de los niños consideraron que los padres tienen toda la autoridad para violentar a sus hijos y su conducta no resulta cuestionable. En otro extremo aparece el $13 \%$ de los niños que no sólo cuestionan la autoridad paterna, sino que consideran la demanda legal a los padres cómo una alternativa de defensa.

Al preguntar quién podría impedir el maltrato hacia los niños se pudo determinar que un porcentaje de $21 \%$ consideraron que nadie podría cuestionar la autoridad paterna. El $30 \%$ piensan que podían ellos mismos cuestionarla y un porcentaje de $20 \%$ sugieren acudir a un familiar. Solamente el $14.3 \%$ recurrirían a entidades públicas y un $10 \%$ al contexto escolar. La Tabla 3 muestra el comportamiento por edades.

Tabla 3

Cuestionamiento de la autoridad paterna

\begin{tabular}{ccc}
\hline Edad & $\begin{array}{c}\text { Cuestionan la } \\
\text { autoridad paterna }\end{array}$ & $\begin{array}{c}\text { No cuestionan la } \\
\text { autoridad paterna }\end{array}$ \\
$6-8$ & $62.1 \%$ & $34.5 \%$ \\
$9-11$ & $84.2 \%$ & $15.8 \%$ \\
$12-14$ & $100 \%$ & \\
\hline
\end{tabular}

Fuente: Los autores

El análisis respecto a la clase social determinó que el $80 \%$ de los niños de clase social alta consideran cuestionable la autoridad paterna y un $20 \%$ la consideran incuestionable. Un $60 \%$ consideran que ellos podrían cuestionar directamente dicha autoridad hablando con los padres.

c) ¿Quién cuestiona la autoridad paterna?

Ante la pregunta de quiénes podrían ayudar o a quién se podría acudir, el comportamiento por edades mostró la siguiente tendencia, ver Tabla 4 
Tabla 4

Porcentaje de entidades y personas que cuestionan la autoridad paterna

\begin{tabular}{ccccc}
\hline Edad & Familiares & Colegio & Entidades Públicas & Niños \\
$6-8$ & $24.1 \%$ & $13.8 \%$ & $10.3 \%$ & $37.9 \%$ \\
$9-11$ & $36.8 \%$ & $21.1 \%$ & $15.8 \%$ & $52.6 \%$ \\
$12-14$ & $33.3 \%$ & $66.7 \%$ & -- & -- \\
\hline
\end{tabular}

Fuente: Los autores

\section{d) Castigos}

Los niños en general consideraron alternativas de castigo, que no involucran la violencia física. El $48.3 \%$ de los niños entre $6-8$ años consideraron que el diálogo podría ser la alternativa de reflexión ante las fallas de los niños. Se contemplan otros castigos cómo reparadores (20.7\%), quitar privilegios $(13.8 \%)$ y expiatorios $(3.4 \%)$. Los niños de 9-11 años proponen en un 47.4\% el diálogo o un regaño. Sin embargo el $42.1 \%$ no proponen castigos diferentes al castigo físico. Los castigos reparadores fueron muy poco nombrados por este grupo de niños (Ver Tabla 5).

Tabla 5

Tipos de castigo

\begin{tabular}{|c|c|c|c|c|c|}
\hline Edad & Diálogo & Reparadores & Expiatorios & Castigo físico & Quitar privilegios \\
\hline $6-8$ & $48.3 \%$ & $20.7 \%$ & $3.4 \%$ & $27.6 \%$ & \\
\hline $9-11$ & $47.4 \%$ & $5.2 \%$ & $5.3 \%$ & $42.1 \%$ & \\
\hline $12-14$ & $50 \%$ & & & $33.3 \%$ & $17 . \%$ \\
\hline
\end{tabular}

Fuente: Los autores

Cabe destacar que en el análisis por clase social el 70\% de los niños de clase alta consideraron el diálogo como una alternativa ante el castigo físico y un $30 \%$ proponen otros castigos entre los que se encuentra el regaño.

\section{e) Derechos}

El 69\% de los niños no saben qué significa la palabra Derechos. Solamente el 31\% intentaron definirlo a través de un ejemplo. El derecho a la educación fue considerado cómo uno de los principales derechos $(27.6 \%)$ así como el derecho a jugar (24.1\%), la alimentación (20.7\%), el derecho a tener una familia $(17.2 \%)$ y a no ser maltratado. De los niños de 9-11 el 47.4\% trata de definir el concepto de derecho a través de ejemplos y el $52.6 \%$ no saben qué es un derecho. El 21.1\% los define como inherente al ser humano y universal mientras que $10.5 \%$ lo definen como un privilegio. En este grupo de edad se reconocen los siguientes derechos: a la educación (68.4\%), seguido por otros derechos como, el amor o la familia (42.1\%), jugar y alimentarse (21.1\%), libertad de expresión $15.8 \%$ y un $10.5 \%$ a no ser maltratados.

Respecto a la clase social, el $45 \%$ de niños de clase alta, no conocen el concepto y no lo pueden definir. Es importante resaltar que el $40 \%$ de los niños de clase alta consideran la familia como uno de los derechos más importantes. En tanto que el derecho a la educación (29.4\%) y el derecho a no ser maltratado con $17.6 \%$ fueron los que los niños de clase baja señalaron cómo más importantes, teniendo en cuenta que el $67.6 \%$ no conocen el concepto de derecho.

\section{f) Discusión}

Los resultados obtenidos permiten analizar algunos aspectos importantes acerca de la comprensión de los derechos por parte de los niños. En primer lugar se destaca la dificultad que representa en los niños, menores de 11 años, definir el concepto de derecho. 69\% de la muestra total no pudo definirlo o determinar su significado. Este dato es muy importante porque cuestiona el uso de este término en escenarios educativos, donde se difunden los derechos de los niños, pero no se toma en cuenta qué concepto tienen los niños de sus propios derechos y qué significado se le da a la palabra derecho.

Otro resultado interesante se relaciona con el porcentaje de niños que consideran que la autoridad paterna es incuestionable y que los padres tienen todos los derechos 
sobre los hijos. Un $34 \%$ de niños entre 6 y 8 años consideraron este hecho, aunque un porcentaje mayor al $66 \%$ no lo hizo. De otro lado, sólo el $15 \%$ de los niños de 9 11 no cuestionaron la autoridad paterna. Este resultado podría analizarse desde una perspectiva del desarrollo moral que evidentemente aclararía que los niños más pequeños presentan una moral heterónoma y los más grandes (12-14) una moral autónoma (Piaget, 1932; Villegas de Posada, 1998) que les permite ser críticos ante sus padres. Ahora bien, la aceptación total de la autoridad paterna implica que se admite cualquier acto que provenga de ésta. En este caso se justifica incluso el propio maltrato y se genera una preocupación respecto a lo que implica la paternidad dentro de una cultura patriarcal. Otro dato interesante que contradice la perspectiva piagetiana es la tendencia de los niños de 6-8 años a no defender el castigo expiatorio como alternativa de reparación de una falta. Por el contrario, un porcentaje del $48 \%$ considera que lo mejor era el diálogo y un $20 \%$ apuntó a la reparación.

Sin embargo, un dato que puede llegar a preocupar es la insistencia de los niños de todas las edades, de recurrir frecuentemente a la alternativa de castigo físico como parámetro de crianza. Incluso los jóvenes entre 12 y 14 años consideran que esta alternativa puede ser viable como forma de educación de los niños ¿Por qué podrían ser los mismos niños quienes justifican el maltrato físico? Quizás la respuesta está relacionada con la propia experiencia y la consideración que el castigo debe ser equiparable a la gravedad de la falta. Entre mayor la falta, mayor el castigo. Igualmente existe un porcentaje elevado en todas las edades de niños y jóvenes que aceptan el castigo físico por las consecuencias de los actos. Esta investigación, aunque inicial, puede abrir una pregunta respecto a la educación en derechos ¿Es posible que los niños pequeños logren comprender el concepto de derecho y puedan hablar de éste de manera cotidiana? Si la palabra es tan difícil de definir para ellos es posible que el concepto lo sea igualmente.

Es importante reflexionar sobre la importancia de trabajos que permitan en los niños una comprensión del concepto de derechos desde una postura asimilable a cada edad. De otro lado, se debe comenzar a difundir el rechazo generalizado al castigo físico como una forma de crianza. Si se lograra que los niños puedan criticar esta postura y además considerarla inapropiada, podríamos encontrar una salida al eterno ciclo de la violencia.

\section{Anexo A \\ Historia de Juan /Pepita (niños entre 6-11 años)}

Están Juan / Pepita jugando con un balón muy contento (a). De repente el balón le pega a un florero y este se rompe. Ella/él se asustan mucho. Los papás llegan, lo (a) regañan y les pegan duro. Los golpes les hacen daño hasta el punto de no poder mover sus brazos. Los amigos del barrio llegan a jugar con él (ella) pero no puede salir porque le duelen mucho los golpes. El/ella queda muy triste.
Preguntas

¿Tú crees que los papás podían haber actuado de otra manera?

¿Tú crees que Pepita o Juan se merecían el castigo?

¿Hay alguien que pueda prohibirles a los padres golpear a sus hijos?

peguen?

¿Sabes tú que es tener derecho a algo?

¿Has oído hablar de los derechos de los niños?

¿Cuáles son?

¿Cuáles son los más importantes?

¿Cómo puedes tu pedir que se te respeten esos derechos? ¿Los niños pueden hacer algo para que los papás no les

\section{Historia de Juan y Rosario (12-14 años)}

Juan y Rosario tienen 15 años y hace muy poco comenzaron a ser novios. Un día estaban besándose y papá de Rosario los vio. De manera muy violenta les gritó muchas groserías, mandó a su hija a la casa y le pegó varios puños a Juan. Les prohibió volver a verse y ahora tiene a Rosario encerrada en la casa sin poder salir.

\section{Referencias}

Ariès, P. (1987)El niño y la vida familiar en el Antiguo Régimen. Madrid: Taurus.

Baumrind, D. (1978). Reciprocal rights and responsibilities in parent-child relations. Journal of Social Issues 34(2), 179-196.

Benavides, J. (2015). La paradoja de la evolución del concepto de desarrollo. Revista de Psicología Universidad de Antioquia, 7(1), 199-210.

Benavides, J., \& Miranda, S. (2007). Actitud crítica hacia el castigo físico e niños víctimas de maltrato infantil. Universitas Psyhologica, 3(2), 309-318.

Delval, J. (2001). Descubrir el pensamiento de los niños. Madrid: Paidós. XXI.

Delval, J. (1995). El desarrollo humano. Madrid: Siglo

Donova, P., Oñate, X., Bravo, G., \& Rivera, M.T. (2008). Niñez y juventud en situación de riesgo. Una revisión Bibliográfica. Última Década 28, 51-78.

Hart,S. (1991). From property to person status. American Psychologist 46(1), 53-59.

Melton, G. (1980). Children's concepts of their rights. Journal of Clinical Child Psychology, fall, 186-190.

Piaget, J. (1932). El criterio moral en el niño. Barcelona: Fontanella. 
Rogers, C. y Wrightsman, L. (1978). Attitudes toward Children's Rights: Nurturance or Self determination. Journal of Social Issues 34(2), 59-68.

Takanishi, R. (1978). Childhood as a social issue: Historical roots of contemporary child advocacy movements. Journal of Social Issues. 34(2), 8-25.

Villegas de Posada, M.C. (1998). Influencia de Piaget en el estudio del desarrollo moral. Revista Latinoamericana de Psicología, 30(2), 223-232.
W H O . $\quad\left(\begin{array}{llll}2 & 0 & 1 & 4\end{array}\right)$. T o m a d o d e http://www.paho.org/nutricionydesarrollo/?p=4023 (abril 152016).

Worsfold, V. (1974). A Philosophical justification for children's rights Harvard Educational Review. 44(1), 142157 